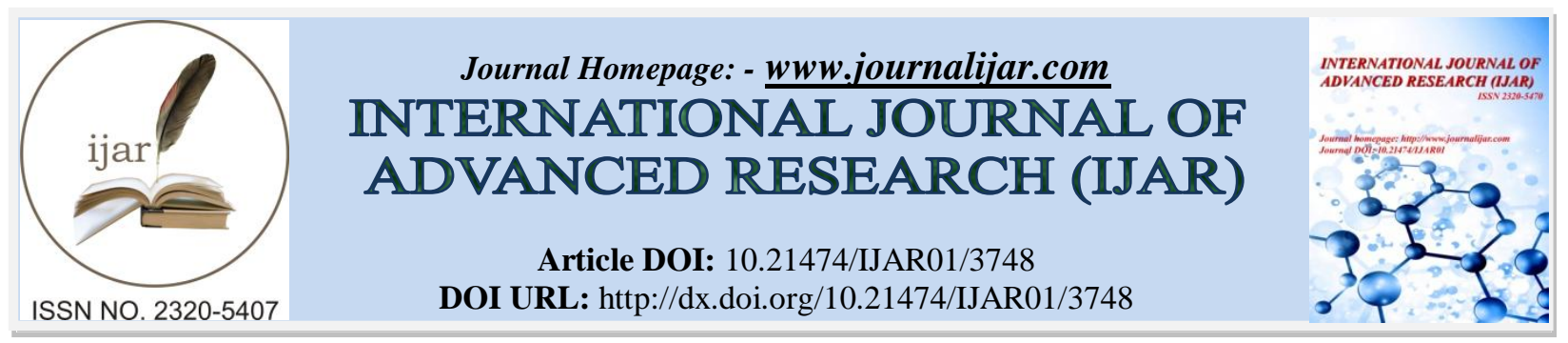

RESEARCH ARTICLE

\title{
PULMONARY ALVEOLAR MICROLITHIASIS: CLINICAL AND RADIOPATHOLOGICAL REVIEW.
}

\author{
Dr. Tushar Sahasrabudhe and Dr. Sowmya Attipatla.
}

Department of Pulmonary medicine, Dr. D.Y.Patil Medical college and Research institute, Pimpri, Pune.

\section{Manuscript Info}

Abstract

Manuscript History

Received: 16 January 2017

Final Accepted: 03 February 2017

Published: March 2017

Copy Right, IJAR, 2017,. All rights reserved.

\section{Introduction:-}

Pulmonary alveolar microlithiasis (PAM) is a rare and slowly progressive diffuse chronic lung disease characterized by deposition of small calcium phosphate spherules within the alveolar cavity.(1) The etiology of the disease is still unknown and is supposed to be due to defect in the inherited local enzyme that is responsible for calcium deposition.(2)The disease is usually seen from birth up to 40 years of age.(1,3) Clinically, the disease course is different. Most of the patients are asymptomatic or having very mild symptoms and majority of patients either having normal or restrictive pulmonary function tests. $(1,3)$

\section{Case:-}

We report a case of 44-year-old male, tailor by occupation who presented with breathlessness on exertion for 3 years. Initially, grade I MMRC dyspnea progressed to grade II MMRC on presentation, over 3 years. Associated with mild cough and scanty expectoration which was gradually progressive, non-foul smelling, non-blood tinged. No history of fever/chest pain.

Personal history-Smoker for 10 years,1-2 cigarettes/day, stopped 5years back. Also, had a history of cement dust exposure from a nearby cement factory for 25-30 years. No significant family history.

On general examination vitals, stable, clubbing grade 2 observed. No pallor, icterus, cyanosis, lymphadenopathy or edema. Systemic examination: Upper respiratory tract normal.

On inspection, chest bilaterally symmetrical, trials sign negative. No scars, sinuses or dilated veins seen, movements bilaterally equal. Palpation: Inspectory findings confirmed, no local rise of temperature or tenderness, tactile vocal fremitus equal on both sides. Percussion: Resonant note all over lung fields. On auscultation: Late fine inspiratory crepitations heard over interscapular, infrascapular and infraaxillary areas. Vocal fremitus: equal on both sides.

Rest of the systems are normal.

On chest x-ray (Fig-1) diffuse, dense, large calcifications noted in bilateral lung fields. HRCT chest (Fig-2,3) revealed dense, large parenchymal calcifications in apico-posterior and anterior segments of bilateral upper lobes, thick septal calcifications in rest of the lung fields, predominantly in the basal segments of the bilateral lower lobes.Spirometry showed restrictive pattern. Bronchoscopy showed fibrosis in the secondary bronchi Bronchoscopic guided lung biopsy was done. 
Histopathological examination (Fig-4) of the lung section revealed calcified microlith in the intra-alveolar spaces. Special stain -Von kossa stain (Fig-5) positive confirmatory of pulmonary alveolar microlithiaisis.

\section{Treatment:-}

Treatment with corticosteroids and symptomatic treatment was given and on follow up visits, patient improved symptomatically.

\section{Discussion:-}

PAM is a rare chronic parenchymal lung disease often diagnosed incidentally in asymptomatic patients or become symptomatic between 3 rd and $4^{\text {th }}$ decades $(1,3,4,5)$. Basically characterized by numerous small calculi (denominated calciferites, calcospherites or microlites) within air spaces.(5)Calculi are round or ovoid in shape and range from 0.01 to $2.8 \mathrm{~mm}$.(2) Studies confirmed that the disease is caused by autosomal recessive inheritance of a mutation in the gene (SCL34A2 gene) on chromosome 4,that produces the protein that is the only known sodium-dependent phosphate transporter in the lungs. $(4,6)$ As a result of this mutation, the protein is unable to transport phosphorus ions from the alveolar space into type II pneumocytes, and this failure leads to the development of calcospherites in the alveolar space.(6) At clinical presentation patients usually demonstrate a lung disorder with restrictive pattern as seen in our case. Adult patients commonly show progressive deterioration of the pulmonary function and death usually occurs in mid-life because of respiratory failure associated with cor pulmonale.(5)

Plain chest x-ray usually shows diffuse bilateral symmetric micronodular calcification (sandstorm) mainly in middle and lower zones; apical bullae and black pleural lines. HRCT thorax remains the imaging technique of choice to diagnose PAM. Findings include ground glass opacity (most common), sub-pleural calcification, confluent and diffuse calcified nodules and dense consolidation. Septal thickening, 'black pleura' sign due to sub-pleural cyst and crazy paving pattern have also been described. $(4,5)$ Although the imaging can help in diagnosing the disease but the lung biopsy is the best for definitive diagnosis.(4)Measurement of surfactant protein A (SP-A) and surfactant protein D (SP-D) can be considered as an alternative in monitoring disease activity.(4,5) Differential considerations include sarcoidosis, pneumoconiosis, pulmonary hemosiderosis, amyloidosis, miliary tuberculosis, and metastatic pulmonary calcifications associated with chronic renal failure.(6)There is no effective treatment for PAM. Use of disodium etidronate in the treatment of PAM is associated with mixed results. Lung transplantation is the only hope for end-stage PAM. (4) 


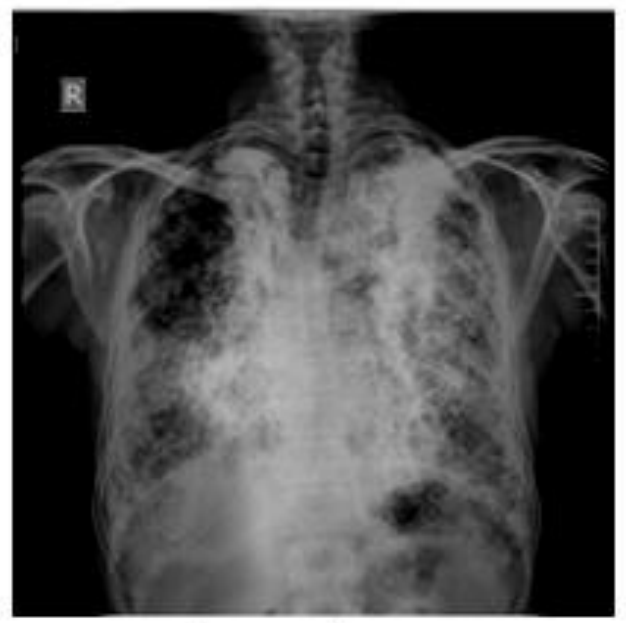

Fig-1

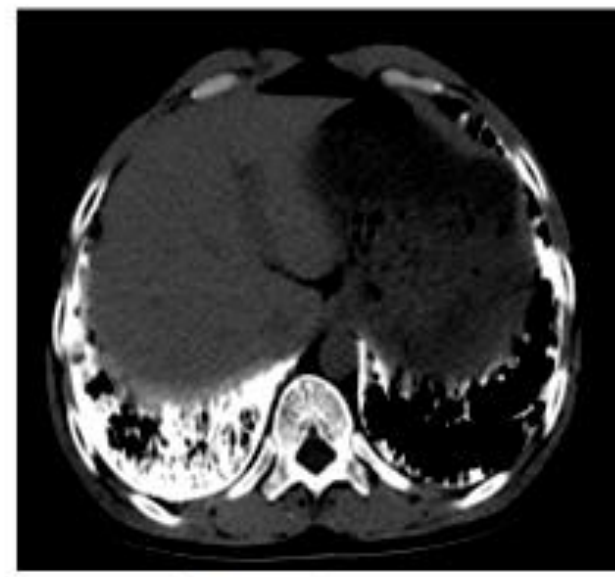

Fig-3

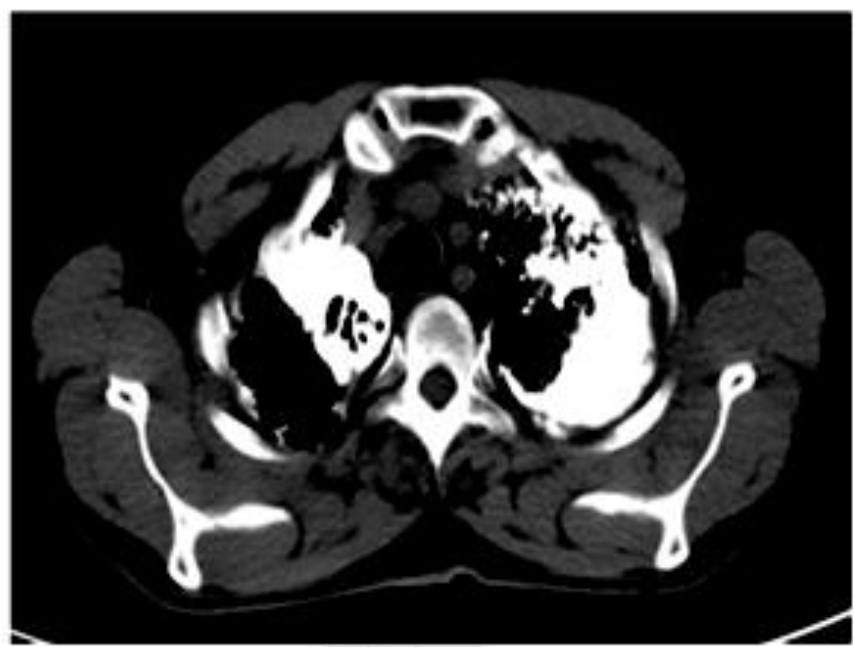

Fig-2

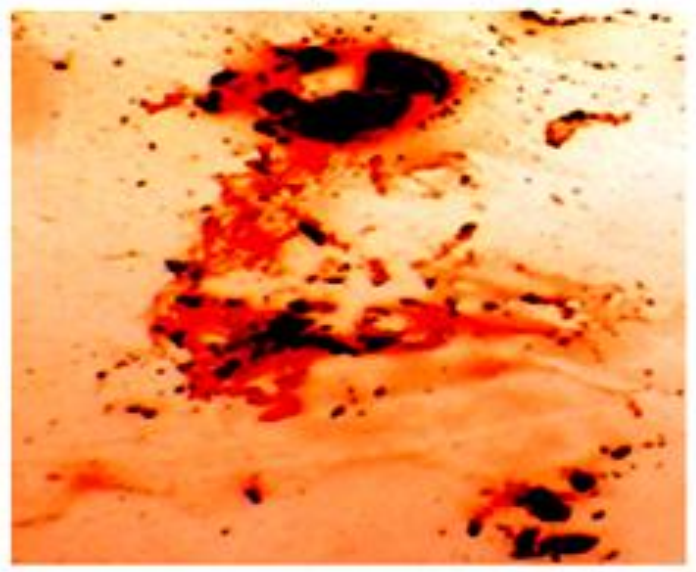

Fig-4

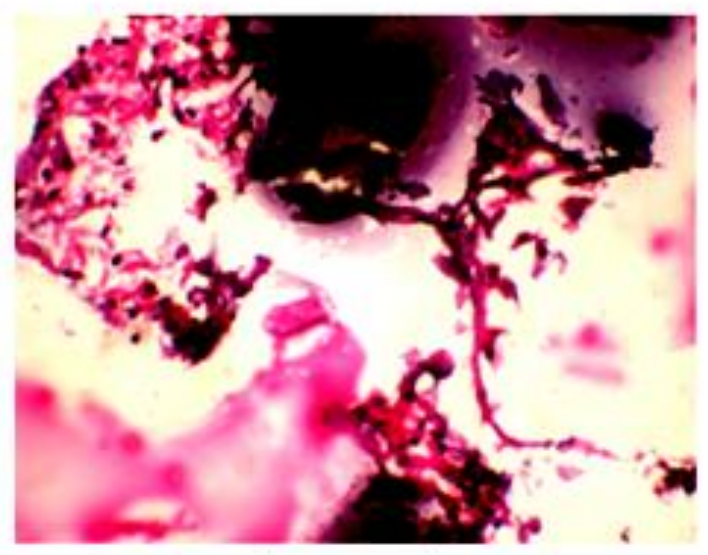

Fig-5 


\section{References:-}

1. Rebeen R. Saeed, Kosar M. Ali. A 55 years old man with pulmonary alveolar microlithiasis, Lung India,Vol 31,Issue 3, Jul - Sep 2014

2. Salvatore Mariotta, Alberto Ricci, Maria Papale, Francesca De Clementi, Bruno Sposato etal. Pulmonary alveolar microlithiasis: report on 576 cases published in the literature, sarcoidosis vasculitis and diffuse lung diseases 2004; 21: 173-181

3. 3.Asa Lina M. Jonsson, Ulf Simonsen, Ole Hilberg and Elisabeth Bendstrup, Pulmonary alveolar microlithiasis: two case reports and review of the literature. Eur Respir Rev $2012 ; 21: 125,249-256$

4. 4.Debaprasad Chakrabarti, Prabhat Debbarma, Amrit Kumar Bhattacharyya, Pulmonary Alveolar Microlithiasis: A Clinicoradiological Dissociation. Indian Journal of Clinical Practice, Vol. 24, No. 1, June 2013

5. 5.Guilherme Abdalla, EdsonMarchiori, Glaucia Zanetti, AntonioMucillo, etal. Pulmonary Alveolar Microlithiasis: A Case Report with emphasis on Imaging Findings. Case Reports in Medicine Volume 2010

6. Nasir A. Siddiqui, MD, Carl R. Fuhrman, MD. Best Cases from the AFIP:Pulmonary Alveolar Microlithiasis. RadioGraphics 2011; 31 